\title{
NOTE ON POLYHARMONIC FUNCTIONS ${ }^{1}$
}

\section{R. J. DUFFIN ${ }^{2}$ AND Z. NEHARI}

Let $\Delta$ denote the $m$-dimensional Laplace operator $\sum_{\mu=1}^{m} \partial^{2} / \partial x_{\mu}^{2}$ and let the positive number $r$ be defined by $r^{2}=\sum_{\mu=1}^{m} x_{\mu}^{2}$. If $u(P)$ is a harmonic function in the sphere $\bar{P} \bar{O}=r<R-$ i.e., $u(P)$ is continuous and has continuous first and second derivatives for $r<R$ and satisfies there the equation $\Delta u=0$-and if, moreover, $u(P)>0$ for $r<R$, then, according to a classical result (Harnack's inequality)

$$
u(P) \leqq u(0) \frac{R^{m-2}(R+r)}{(R-r)^{m-1}} .
$$

It is the purpose of this note to show that there exists an inequality of the same general character as (1) if the hypothesis that $u(P)$ be harmonic is replaced by the considerably weaker assumption that $u(P)$ be a polyharmonic function of order $n(n>1)$. The latter expression refers to a function which is continuous, has continuous derivatives up to the order $2 n$, and is a solution of $\Delta^{(n)} u=0$, where $\Delta^{(k)}$ is defined as $\Delta\left(\Delta^{(k-1)}\right)$. Our precise result is the following:

If $u(P)$ is a non-negative polyharmonic function of order $n$ in the $m$-dimensional sphere $\bar{O} \bar{P}=r<R$, then

$$
u(P) \leqq A R^{m-2} \frac{(R+r)}{(R-r)^{m-1}}
$$

where

$$
A=m ! 2^{n+m-2} \sum_{\nu=0}^{n-1} c_{\nu}\left|\Delta^{(\nu)} u(0)\right| R^{2 \nu}
$$

and

$$
c_{\nu}^{-1}=2^{\nu} \nu ! m(m+2) \cdots(m+2 \nu-2) .
$$

The proof of (2) will be based on the fact [1] that a function $u(P)$ which is polyharmonic of order $n$ in $r<R$ has a representation

$$
u(P)=\sum_{\nu=0}^{n-1} u_{\nu}(P) r^{2 \nu}, \quad r<R,
$$

Received by the editors April 15, 1960.

1 This research was supported by the United States Air Force Office of Scientific Research.

2 Visiting Professor at the Dublin Institute for Advanced Studies. 
where the functions $u_{\nu}(P)$ are harmonic for $r<R$. In order to facilitate the writing we shall assume that $u(P)$ is regular in $r \leqq R$. No generality is lost by this assumption, since it can be made to hold by the preliminary coordinate transformation $r \rightarrow r(1-\epsilon)$, where $\epsilon$ is a small positive number, and $\epsilon$ can be made to tend to zero in the final result. If we regard the right-hand side of (5) as a polynomial in $r^{2}$ and set

$$
v\left(P ; \rho^{2}\right)=\sum_{\nu=0}^{n-1} u_{\nu}(P) \rho^{2 \nu},
$$

it follows from the Lagrange interpolation formula that

$$
u(P)=\sum_{\nu=1}^{n} v\left(P ; R_{\nu}^{2}\right) \frac{F_{\nu}\left(r^{2}\right)}{F_{\nu}\left(R_{\nu}^{2}\right)},
$$

where

$$
F_{\nu}\left(r^{2}\right)=\prod_{k=1 ; k \neq \nu}^{n}\left(R_{\nu}^{2}-r^{2}\right),
$$

and the $R_{\nu}$ are positive numbers which satisfy $r<R_{1}<R_{2}<\ldots$ $<R_{n-1}<R_{n}=R$ and are otherwise arbitrary.

For a fixed value of $\rho$, the function $v\left(P ; \rho^{2}\right)$ is harmonic for $r \leqq R$. If $P$ is such that $\bar{O} \bar{P}=\rho$, a comparison of (5) and (6) shows that $u(P)=v\left(P ; \rho^{2}\right)$, and it follows from our hypotheses that $v\left(P ; \rho^{2}\right) \geqq 0$. But $v\left(P ; \rho^{2}\right)$ is a harmonic function of $P$, and the maximum principle shows therefore that $v\left(P ; \rho^{2}\right) \geqq 0$ for all $P$ such that $\bar{O} \bar{P} \leqq \rho$. Since $R_{\nu}^{2}>r^{2}$, the expressions $v\left(P ; R_{\nu}^{2}\right)$ in (7) will accordingly all be nonnegative. By (8), the value of $F_{\nu}\left(R_{\nu}^{2}\right)$ is positive if $\nu$ is odd, and negative if $\nu$ is even. We may thus conclude from (7) that

$$
u(P) \leqq \sum_{\nu=1}^{n} v\left(P ; R_{\nu}^{2}\right) \frac{F_{\nu}\left(r^{2}\right)}{F_{\nu}\left(R_{v}^{2}\right)},
$$

where the prime indicates that the summation is to be extended only over the odd values of $\nu$.

We now set

$$
R_{\nu}^{2}=r^{2}+\frac{\nu}{n}\left(R^{2}-r^{2}\right) .
$$

By (8), we then have $F_{\nu}\left(r^{2}\right)=\nu^{-1} n^{1-n} n !\left(R^{2}-r^{2}\right)^{n-1}$, and $F_{\nu}\left(R_{\nu}^{2}\right)$ $=(\nu-1) !(n-\nu) ! n^{1-n}\left(R^{2}-r^{2}\right)^{n-1}$. Inequality (9) will thus reduce to

$$
u(P) \leqq \sum_{\nu=1}^{n}\left(\begin{array}{l}
n \\
\nu
\end{array}\right) v\left(P ; R_{\nu}^{2}\right),
$$


where $R_{\nu}^{2}$ is defined by $(10)$. Since $v\left(P ; R_{\nu}^{2}\right)$ is a non-negative harmonic function for $\bar{O} \bar{P} \leqq R_{\nu}$, it follows from (1) and (6) that

$$
v\left(P ; R_{\nu}^{2}\right) \leqq\left(\sum_{k=0}^{n-1} u_{k}(0) R_{\nu}^{2 k}\right) \frac{R_{\nu}^{m-2}\left(R_{\nu}+r\right)}{\left(R_{\nu}-r\right)^{m-1}} .
$$

Setting

$$
B=\sum_{k=0}^{n-1}\left|u_{k}(0)\right| R_{\nu}^{2 k}
$$

and observing that $\sum_{k=0}^{n-1} u_{k}(0) R_{\nu}^{2 k} \leqq B$ and

$$
\frac{R_{\nu}^{m-2}\left(R_{\nu}+r\right)}{\left(R_{\nu}-r\right)^{m-1}}<\frac{R^{m-2}(R+r)^{m}}{\left(R_{\nu}^{2}-r^{2}\right)^{m-1}}=\left(\frac{n}{\nu}\right)^{m-1} \frac{R^{m-2}(R+r)}{(R-r)^{m-1}},
$$

we obtain

$$
u(P) \leqq B \frac{R^{m-2}(R+r)}{(R-r)^{m-1}} \sum_{\nu=1}^{n}\left(\frac{n}{\nu}\right)^{m-1}\left(\begin{array}{l}
n \\
\nu
\end{array}\right) .
$$

If $k$ is a positive integer, we have $n / \nu<(k+1)(n+k) /(\nu+k)$, whence

$$
\left(\frac{n}{\nu}\right)^{m-1}<m ! \frac{(n+1)(n+2) \cdots(n+m-1)}{(\nu+1)(\nu+2) \cdots(\nu+m-1)},
$$

and therefore

$$
\left(\frac{n}{\nu}\right)^{m-1}\left(\begin{array}{l}
n \\
\nu
\end{array}\right)<m !\left(\begin{array}{c}
n+m-1 \\
\nu+m-1
\end{array}\right) .
$$

Accordingly,

$$
\sum_{\nu=1}^{n}\left(\frac{n}{\nu}\right)^{m-1}\left(\begin{array}{l}
n \\
\nu
\end{array}\right)<m ! \sum_{\mu=1}^{n+m-1}\left(\begin{array}{c}
n+m-1 \\
\mu
\end{array}\right),
$$

where the prime on the right-hand side indicates that the summation is to be extended only over indices $\mu$ such that $\mu \equiv m(\bmod 2)$. Since the value of this sum is $2^{n+m-2}$, we thus conclude from (12) that

$$
u(P)<2^{n+m-2} m ! B \frac{R^{m-2}(R+r)}{(R-r)^{m-1}} .
$$

In view of (11), (5), and the fact that, for a harmonic function $w(P)$,

$$
\Delta^{(k)}\left(r^{2 k} w\right)_{P=0}=2^{k} k ! m(m+2) \cdots(m+2 k-2) w(0)
$$

and $\Delta^{(k)}\left(r^{2 \mu} w\right)_{P=0}=0$ for $\mu \neq k$, this completes the proof of inequality (2). 
For any special value of $n$, a more accurate inequality can be obtained by evaluating the sum on the right-hand side of (12) and avoiding the rather crude estimate (13). For instance, if $n=2-$ i.e., in the case of a biharmonic function $u(P)$ - the summation in (12) reduces to the term corresponding to $\nu=1$. A positive biharmonic function will therefore be subject to the inequality

$$
u(P) \leqq 2^{m}[u(0)+2 m|\Delta u(0)|] \frac{R^{m-2}(R+r)}{(R-r)^{m-1}} .
$$

It may be pointed out that, as the example $u(P)=\bar{P} \bar{Q}^{2} u_{0}(P)$, $\Delta u_{0}=0, u_{0}(P) \geqq 0$ shows, a non-negative polyharmonic function $u(P)$ may vanish at any point $Q$ in the sphere $r<R$. Hence, there does not exist an analogue for polyharmonic functions of Harnack's "lower" inequality for harmonic functions.

As an application of the inequality (2) we prove the following result of Nicolesco $[2 ; 3]$.

If $u(P)$ is non-negative and polyharmonic of order $n$ at all finite points $P$ of the m-dimensional Euclidean space, then $u(P)$ reduces to a polynomial of order $2 n-2$ in the Cartesian coordinates $x_{1}, \cdots, x_{m}$.

By assumption, (2) holds for all $r$ and $R$ such that $0 \leqq r<R<\infty$. Setting, in particular, $R=2 r$, we obtain $u(P)<A C_{1}$, where $C_{1}$ is a constant. By (3), $A<C_{2} r^{2 n-2}$, and thus

$$
0 \leqq u(P) \leqq C r^{2 n-2},
$$

where $C$ is again a constant. The functions $u_{\nu}(P)$ in (5) are harmonic for all finite non-negative values of $r$, and we may therefore expand them into series

$$
u_{\nu}(P)=\sum_{k=0}^{\infty} r r^{k} S_{k}^{(\nu)},
$$

where the $S_{\boldsymbol{k}}^{(\nu)}$ are spherical harmonics of order $k$ and do not depend on $r$. Hence,

$$
u(P)=\sum_{k=0}^{\infty} r^{k}\left(\sum_{\nu=0}^{n} r^{2 v} S_{k}^{(\nu)}\right) .
$$

If $S_{k}$ is any spherical harmonic of order $k$, it follows from the orthogonality properties that

$$
\int_{U_{m}} u(P) S_{k} d \omega_{m}=r^{k} \sum_{\nu=0}^{n-1} A_{k}^{(\nu)} r^{2 \nu}
$$

where $U_{m}$ is the unit sphere in $m$-space, $d \omega_{m}$ the area element on $U_{m}$, and 


$$
A_{k}^{(p)}=\int_{U_{m}} S_{k}^{(\nu)} S_{k} d \omega_{m} .
$$

If $A_{k}$ is a constant such that $\left|S_{k}\right|<A_{k}$, it follows from (14) that

$$
\left|\int_{U_{m}} u(P) S_{k} d \omega_{m}\right| \leqq C A_{k} \Omega_{m} r^{2 n-2},
$$

where $\Omega_{m}$ is the area of $U_{m}$. Hence

$$
\left|r^{k} \sum_{\nu=0}^{n-1} A_{k}^{(\nu)} r^{2 v}\right| \leqq C_{3} r^{2 n-2},
$$

where $C_{3}$ is another constant. If $k>2 n-2$, this shows that

$$
\lim _{r \rightarrow \infty}\left|\sum_{\nu=0}^{n-1} A_{k}^{(v)} r^{2 v}\right|=0
$$

and we must therefore have $A_{\mathfrak{k}}^{(\nu)}=0$ for $\nu=0, \cdots, n-1$ and $k>2 n$ -2 . Since $S_{k}$ was an arbitrary spherical harmonic of order $k$, it follows that the functions $S_{\boldsymbol{k}}^{(\nu)}$ vanish identically. The inequality (16) shows, moreover, that all $A_{k}^{(v)}$ for which $k+2 \nu>2 n-2$ are necessarily zero. The corresponding spherical harmonics $S_{\boldsymbol{k}}^{(\nu)}$ will therefore also vanish identically. Accordingly, the expansion (15) reduces to a polynomial in $r$ of order not exceeding $2 n-2$, and the result follows.

We finally show that the representation (7) provides a simple means of obtaining the Poisson formula analogue for polyharmonic functions without the explicit knowledge of the Green's function. We choose a small positive number $\epsilon$ such that $n \epsilon<R^{2}-r^{2}$ and set $R_{\nu}^{2}=R^{2}-\epsilon \nu$. If $F_{\nu}\left(r^{2}\right)$ is the expression (8), we then have

$$
\left(R_{\nu}^{2}-r^{2}\right) F_{\nu}\left(r^{2}\right)=\prod_{k=1}^{n}\left(R^{2}-r^{2}-k \epsilon\right)
$$

and

$$
F_{\nu}\left(R_{\nu}^{2}\right)=(-1)^{n-\nu} \epsilon^{n-1}(\nu-1) !(n-\nu) !
$$

The function $v\left(P ; R_{\nu}^{2}\right)$ is harmonic for $r \leqq R_{\nu}$ and coincides with $u(P)$ for $r=R_{\nu}(r=\overline{O P})$. It may therefore be represented by the Poisson integral

$$
\frac{R_{\nu}^{m-2}\left(R^{2}-r^{2}\right)}{\Omega_{m}} \int_{U_{m}} \frac{u\left(P_{\nu}\right) d \omega_{m}}{\left(R_{\nu}^{2}-2 r R_{\nu} \cos \gamma+r^{2}\right)^{m / 2}},
$$

where $\Omega_{m}$ is the surface area of the $m$-dimensional unit sphere $U_{m}$, 
$d \omega_{m}$ is the corresponding surface element, and $\gamma$ is the angle between the vectors $O P$ and $O P_{\nu}\left(\overline{O P}_{\nu}=R_{v}\right)$. In view of (7), (17), and (18), we thus have

$u(P)=\frac{\prod_{k=1}^{m}\left(R^{2}-r^{2}-k \epsilon\right)}{(n-1) ! \epsilon^{n-1} \Omega_{m}} \int_{U_{m}}\left[\sum_{\nu=1}^{n}(-1)^{n-\nu}\left(\begin{array}{l}n-1 \\ \nu-1\end{array}\right) \frac{R_{\nu}^{m-2} u\left(P_{\nu}\right)}{k\left(R_{\nu}\right)}\right] d \omega_{m}$, where $k\left(R_{\nu}\right)=\left(R_{\nu}^{2}-2 r R_{\nu} \cos \gamma+r^{2}\right)^{m / 2}$.

If $F\left(\rho^{2}\right)$ is a function having $n-1$ continuous derivatives at $\rho^{2}=R^{2}$, we have

$$
\begin{aligned}
\phi & \equiv \sum_{\nu=1}^{n}(-1)^{n-\nu}\left(\begin{array}{l}
n-1 \\
\nu-1
\end{array}\right) F\left(R_{\nu}^{2}\right) \\
& =\sum_{\nu=0}^{n-1}(-1)^{n-\nu-1}\left(\begin{array}{c}
n-1 \\
\nu
\end{array}\right) F\left(R_{\nu+1}^{2}\right) \\
& =\sum_{\nu=0}^{n-1}(-1)^{\nu}\left(\begin{array}{c}
n-1 \\
\nu
\end{array}\right) F\left(R_{n-\nu}^{2}\right) \\
& =\sum_{\nu=0}^{n-1}(-1)^{\nu}\left(\begin{array}{c}
n-1 \\
\nu
\end{array}\right) F\left(R^{2}-\nu \epsilon\right),
\end{aligned}
$$

and therefore

$$
\lim _{\epsilon \rightarrow 0} \frac{\phi}{\epsilon^{n-1}}=\frac{\partial^{n-1} F\left(R^{2}\right)}{\partial\left(R^{2}\right)^{n-1}} .
$$

Accordingly, a function $u(P)$ which is polyharmonic of order $n$ in the $m$-dimensional sphere $r<R$, and has continuous partial derivatives up to the order $n-1$ in $r \leqq R$, has the representation

$$
u(P)=\frac{\left(R^{2}-r^{2}\right)^{n}}{(n-1) ! \Omega_{m}} \int_{U_{m}} \frac{\partial^{n-1}}{\partial\left(R^{2}\right)^{n-1}}\left[\frac{R^{m-2} u\left(P^{\prime}\right)}{\left(R^{2}-2 r R \cos \gamma+R^{2}\right)^{m / 2}}\right] d \omega_{m} .
$$

For biharmonic functions $(n=2)$ an equivalent formula was found by Lauricella and Volterra (quoted in [3]).

\section{REFERENCES}

1. E. Almansi, Sull'integrazione dell'equazione differenziale $\Delta^{2 u}=0$, Annali di matematica Serie III vol. II (1899) pp. 1-59.

2. M. Nicolesco, Nouvelles contributions dans la théorie des fonctions polyharmoniques, Bull. Math. Soc. Sci. Math. Phys. R.P. Roumaine vol. 37 (1935) p. 100.

3. - Les fonctions polyharmoniques, Paris, Hermann, Actualités Sci. Ind., no. $331,1936$.

Dublin Institute for Advanced Studies and Carnegie Institute of Technology 